Review / Meta-analyses

\title{
Maternal violence experiences and risk of postpartum depression: A meta-analysis of cohort studies
}

\author{
Senmao Zhang ${ }^{\mathrm{a}}$, Lesan Wang ${ }^{\mathrm{a}}$, Tubao Yang ${ }^{\mathrm{a}}$, Lizhang Chen ${ }^{\mathrm{a}}$, Xing Qiu ${ }^{\mathrm{b}}$, Tingting Wang, \\ Letao Chen ${ }^{a}$, Lijuan Zhao ${ }^{a}$, Ziwei Ye ${ }^{a}$, Zan Zheng ${ }^{a}$, Jiabi Qin ${ }^{a}, *$ \\ a Department of Epidemiology and Health Statistics, Xiangya School of Public Health, Central South University, Hunan, China \\ ${ }^{\mathrm{b}}$ School of Nursing, Sun Yat-Sen University, Guangdong, China
}

\section{A R T I C L E I N F O}

\section{Article history:}

Received 10 July 2018

Received in revised form 16 October 2018

Accepted 18 October 2018

Available online 13 November 2018

\section{Keywords:}

Violence

Postpartum depression

Meta-analysis

Cohort study

\begin{abstract}
A B S T R A C T
Background: Most of original studies indicated maternal violence experiences is associated with adverse obstetric outcomes, to date, but it is not clear that the association of maternal violence experiences and the risk of postpartum depression (PPD). We aimed to assess the association between maternal violence experiences and risk of developing PPD by performing a meta-analysis of cohort studies.

Methods: PubMed, Google Scholar, Cochrane Libraries and Chinese databases were searched through December 2017 to identify studies that assessed the association between violence and PPD. Metaanalysis was conducted by the RevMan software and Stata software. Potential heterogeneity source was explored by subgroup analysis and potential publication bias was assessed by Begg's funnel plots and Egger's linear regression test.

Results: Overall, women experiencing any violence events compared with the reference group were at a higher risk of developing PPD (odds ratio $[\mathrm{OR}]=2.04 ; 95 \%$ confidence interval [CI]: 1.72-2.41). Additionally, different types of violence events such as sexual (OR=1.56; 95\%CI: $1.35-1.81)$, emotional $(\mathrm{OR}=1.75 ; 95 \% \mathrm{CI}: 1.61-1.89)$, and physical violence $(\mathrm{OR}=1.90 ; 95 \% \mathrm{CI}: 1.36-2.67)$, as well as domestic $(\mathrm{OR}=2.05 ; 95 \% \mathrm{CI}: 1.50-2.80)$ or childhood violence $(\mathrm{OR}=1.59 ; 95 \% \mathrm{CI}: 1.34-1.88)$ also increased the risk of developing PPD. Relevant heterogeneity moderators have been identified by subgroup analysis. Sensitivity analysis yielded consistent results.

Conclusions: Maternal violence experiences are significantly associated with risk of developing PPD. These finding highlight the necessary to protect women from any types of violence and formulate preventive strategies to promote the maternal mental health.
\end{abstract}

(C) 2018 The Author(s). Published by Elsevier Masson SAS. This is an open access article under the CC BYNC-ND license (http://creativecommons.org/licenses/by-nc-nd/4.0/).

\section{Introduction}

Postpartum depression (PPD) is a common and serious women's mental health problem that is associated with maternal suffering and numerous negative consequences for offspring. The first six months after childbirth may occur a high-risk time for depression [1]. According to previous studies, the prevalence of PPD ranged from $0.9 \%$ to $25.5 \%$ in developed countries, and from $8.2 \%$ to $38.2 \%$ in developing countries using the screen of the Edinburgh Postnatal Depression Scale (EPDS) [2]. Estimates of

\footnotetext{
* Corresponding author: Department of Epidemiology and Health Statistics, Xiangya School of Public Health, Central South University, 110 Xiangya Road, Changsha, Hunan 410078, China.

E-mail address: qinjiabi123@163.com (J. Qin).
}

prevalence ranged from $14.3 \%$ to $19.3 \%$ in China [3,4]. PPD has been identified an acknowledged public health problem [5].

It could increase the risk for multiple adverse outcomes among themself, their partners, infants and families such as lead to selfnegative attitudes, self-harm and even suicidal intention, cause anxiety or depression of partner and even influence the behavioral, cognitive, physical health and social emotional development of their infants and children [1,6-8]. Until now, the etiology of PPD remains unclear, although several risk factors have been identified [2,9], such as history of depression, lack of social support, cesarean delivery and prenatal smoking and so on [10-14]. With the attention of women's mental health, the influence of violence on mental health has attracted the closely attention of researcher. Violence is increasingly becoming recognized as an important public health problem worldwide [15], which may seriously affect the women's mental health, lead to anxiety, depression or posttraumatic stress disorder (PTSD) [16,17]. Previous four systematic reviews and meta-analysis [18-21] suggested that 
maternal violence experiences were significantly associated with the developing of PPD. Of note, these reviews included some case-control studies and cross-sectional studies, which limited the strength and quality of such evidence. Additionally, when exploring the association between violence and PPD, these reviews did insufficient consider the confounding factors and heterogeneity sources, so the risk estimates might be not be precise and robust. Meanwhile, mentioned-above reviews did not given attention to the association between difference types of violence and the risk of PPD.

Furthermore, most of subsequent cohort studies with adequate sample sizes have examined the association in recent years, but the results are inconsistent [22-32]. If these newer studies could be included in the future meta-analysis, it is bound to increase the statistical power. Moreover, our study will to explore the association between the different types of violence and PPD in the same article. Therefore, given the inconsistency of existing literatures and insufficient evidence of primary studies, further an update metaanalysis based on original cohort studies is evidently required.

\section{Methods}

\subsection{Literature search strategy}

We referred to the Preferred Reporting Items for Systematic Reviews and Meta-analyses (PRISMA) guidelines to conduct this meta-analysis [33]. PubMed, Google Scholar, Cochrane Libraries, China Biology Medicine disc, Chinese Scientific Journals Fulltext Database (CQVIP), China National Knowledge Infrastructure (CNKI), and Wanfang Database were searched, with an end date parameter of December 31, 2017, to identify cohort studies assessing maternal violence experiences and risk of PPD. We used and combined the following search terms: "(violence OR abuse OR force OR maltreat OR ill-treat OR tyrannize OR disservice OR negative life event OR adverse event OR traumatic event) AND (postpartum depression OR puerperal depression OR postnatal depression OR depression OR melancholia) AND (cohort study OR prospective study OR follow-up study OR longitudinal study OR incidence study)". Additionally, we also performed a manual search on the reference lists of retrieved articles and recent reviews.

\subsection{Exposure of interest}

In the present review, the exposure of interest was maternal violence experiences which were defined as actual violence, including having been slapped, bitten, kicked, hit, beaten, chocked, threatened with a knife or gun, physical and emotional neglect, or had one used against them or forced into sexual activity [30,34]. Maternal violence experiences could be further classified according to the nature, perpetrators and period of violence occurrence. According to the nature of violence, it could be further divided into sexual violence, emotional violence, and physical violence whereas recently of violence is conceptualized as recent (within the past year) or lifetime (incorporates childhood abuse experiences) [35]. Besides, on the basis of perpetrators and period of violence, it could be further divided into domestic violence [19] (i.e. intimate partner violence perpetrated by a current or former partner and/or violence perpetrated by family member) and childhood violence [36] (i.e. violence occurred when individuals were younger than 18 years of age), respectively. Because previous studies did not explore the association between the different types of violence and the risk of PPD, our study will attempt to estimate these associations.

\subsection{Study selection}

We first performed an initial screening of titles or abstracts. A second screening was based on full-text review. Studies were considered eligible if they met the following criteria: (1) had a cohort study design; (2) the exposure of interest was maternal violence experiences; (3) the outcome of interest was PPD; (4) the determination of outcome were based on reliable techniques such as Edinburgh Postnatal Depression Scale (EPDS), Postpartum Depression Screening Scale (PDSS), Beck Depression Inventory (BDI) and so on; (5) reported relative risks (RRs) and odd ratios (ORs), with corresponding 95\% confidence intervals (CIs) (or data to calculate them).

\subsection{Data extraction and quality assessment}

Two independent authors (SMZ and JBQ) extracted data and assessed study quality. Any disagreements were resolved through discussion among the authors until consensus was reached. Data extraction was performed by using a standardized data collection form. Information was recorded as follows: the first author's name; publication year; geographic region; economic levels (World Bank Income group distribution 2017); study design; number of total samples; assessment method of PPD; PPD assessment time; assessment method of violence; occurrence time of violence (recent vs lifetime); type of violence; reported ORs and their 95\%CIs for the risk of PPD associated with maternal violence experiences; whether the confounding factors were adjusted; and quality score.

In our review, we assessed the study quality of included literatures by using the Newcastle-Ottawa Scale (http://www.ohri. ca/programs/clinical_epidemiology/oxford.asp). Using this scale, each study is judged on eight items, categorized into three groups: the selection of the study groups; the comparability of the groups; and the ascertainment of outcome of interest for cohort studies. Stars awarded for each quality item serve as a quick visual assessment. Stars are awarded such that the highest quality studies can be awarded as many as nine stars. When the study gains seven or more stars, it is considered of higher methodological quality.

\subsection{Statistical analysis}

OR was used as the common measure of association between maternal violence experiences and PPD, and hazard ratios, incidence rate ratios, and RRs were directly considered as OR. The combined ORs and their 95\% CIs were calculated using either fixed-effects models or, in the presence of heterogeneity, randomeffects models. When multiple outcome of violence was reported in the same study, the effect values of the relationship between different types of violence experiences and the risk of postpartum depression were combined with statistical software (such as Stata version 12.0 and Review Manager version 5.3). Homogeneity of effect size across studies was tested by using the $Q$ statistics (significance level at $P<0.10$ ). The $\mathrm{I}^{2}$ statistic (significance level at $\mathrm{I}^{2}>50 \%$ ), which is a quantitative measure of inconsistency across studies, was also calculated [37].

Subgroup analyses according to geographic region, economic levels, assessment method of PPD, PPD assessment time, assessment method of violence, occurrence time of violence, whether the confounding factors were adjusted, and quality score were performed, to assess the potential effect modification of these variables on outcomes. We also conducted a sensitivity analysis to investigate the influence of a single study on the overall risk estimate by omitting one study at a time or excluding the low quality studies. Potential publication bias was assessed by Begg's funnel plots and Begg's linear regression test (significance level at $P<0.10)$ [38].

All statistical analyses were performed using Stata version 12.0 and Review Manager version 5.3. All reported $P$ values were twosided and $P<0.05$ was considered statistically significant, except where otherwise specified. 


\section{Results}

\subsection{Literature search}

We initially searched 508 potentially eligible articles from seven databases. Three additional articles were found from reference lists. Most articles $(n=403)$ were excluded after the first screening based on titles or abstracts because they were duplicates, reviews, or unrelated to our topics. Then, 105 potentially relevant articles were identified, which were carefully assessed by full-text, and 73 articles were further excluded for various reasons. Reasons for not including the other studies were: (i) outcome measures could not be extracted $(n=17)$; (ii) ineligible study design $(n=29)$; (iii) outcome lumping PPD and other diseases $(n=15)$; and (iv) exposed group is inconsistent with our interest $(n=12)$. Finally, we identified 32 eligible articles [15-17,22-32,34-36, 39-53] for this meta-analysis (Fig. 1).

\subsection{Study characteristics}

The characteristics of included literatures, which involved 177,531 participants and were published between 2002 and 2017, were summarized in Table 1 . Among the 32 studies, 11 studies (34.4\%) were conducted in Asia, 9 (28.1\%) in America, 7 (21.9\%) in Oceania, 3 (10.3\%) in Europe and 2 (6.3\%) in Africa. Based on World Bank Income group distribution, 12 studies (37.5\%) belonged to middle and low income countries and 20 studies (62.5\%) belonged to high income countries.

The Edinburgh Postnatal Depression Scale (EPDS) was the most commonly used assessment instrument of PPD (87.5\%), while the Beck Depression Inventory (BDI), the Beck Depression Inventory Fast Screen (BDI-FS), the Patient Health Questionnaire-9 (PHQ-9) and Postpartum Depression Screening Scale (PDSS) only were used in one study respectively. The assessment time of PPD in 20 studies (62.5\%) was conducted within 2 months of postpartum. Eight studies (25\%) used administered questionnaire to assess maternal violence experiences, 12 studies (37.5\%) used standardized/ structured questionnaire or interview and 12 studies (37.5\%) used the violence assessment scale. Meanwhile, according to violence assessment of period, more than half of studies (53.1\%) of violence experiences occurred in recent and other studies (46.9\%) of violence experiences occurred in lifetime.

Additionally, 19 studies (59.4\%) adjusted some potential confounding factors (such as age, education, parity, social support, history of depression, depression during pregnancy and so on), and the remaining studies did not adjusted any factors when estimating the effect. Twenty-nine studies (90.6\%) were considered of higher methodological quality, achieving a quality score $\geq 7$ out of 9; these 29 studies contributed most of study participants. Physical violence experiences in 8 studies were reported, emotional violence experiences in 8 studies, sexual violence experiences in 6 studies, domestic violence experiences in 16 studies, and childhood violence experiences in 5 studies.

\subsection{Quantitative synthesis}

Fig. 2 shows the results from random-effect models combing the ORs between maternal violence experiences and risk of developing PPD. Overall, women who experienced any violence events compared with those who did not experience any violence events, were at a higher risk of developing PPD (OR $=2.04 ; 95 \% \mathrm{CI}$ : 1.72-2.41). However, substantial heterogeneity was found $\left(P=0.000 ; \mathrm{I}^{2}=93.7 \%\right)$.

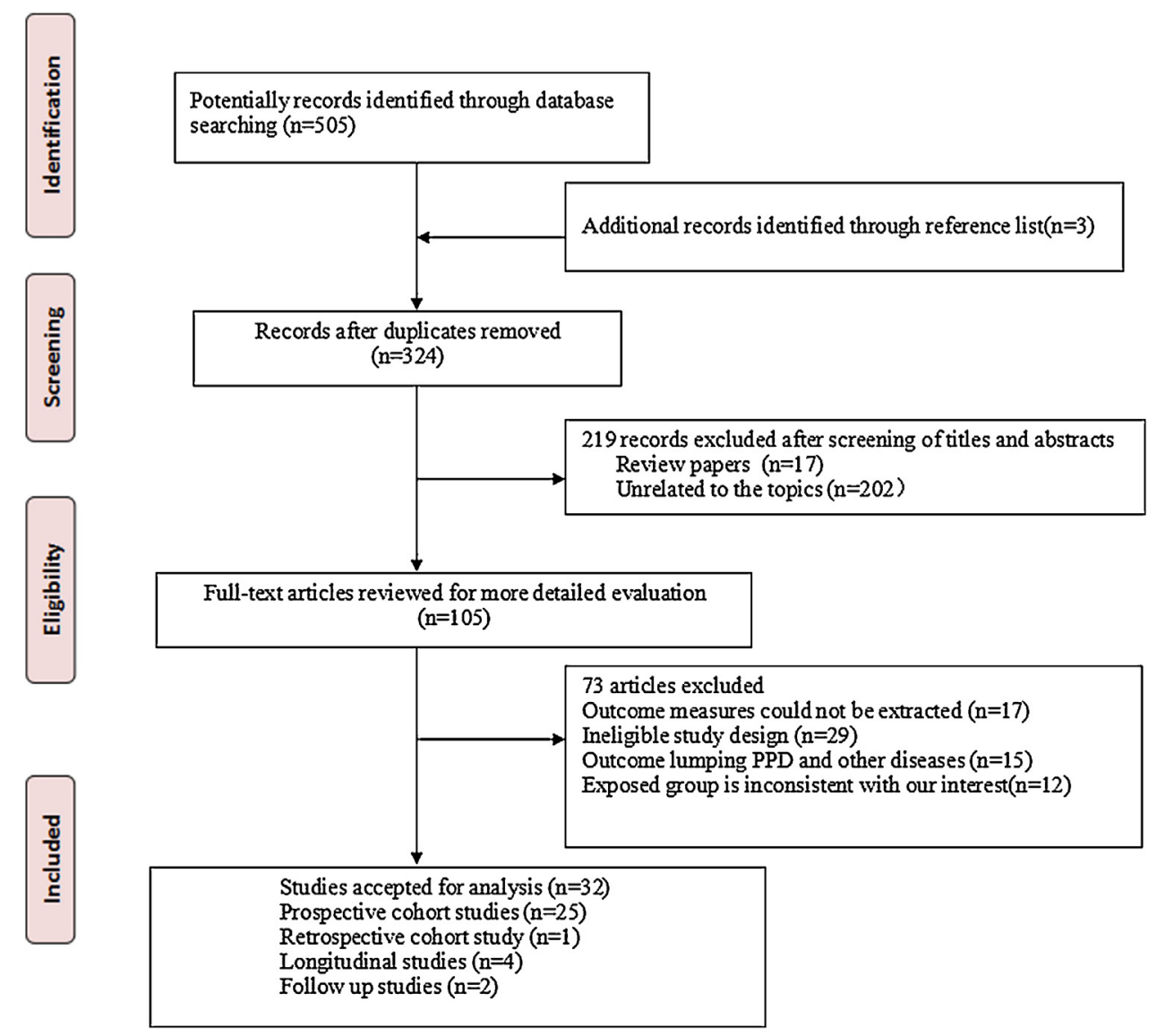

Fig. 1. Flow chart of literature selection. 
Table 1

Characteristics of included studies for the association between violence and risk of PPD.

\begin{tabular}{|c|c|c|c|c|c|c|c|c|c|c|c|}
\hline $\begin{array}{l}\text { First author } \\
\text { (reference) } \\
\text { publication } \\
\text { year }\end{array}$ & $\begin{array}{l}\text { Geographic } \\
\text { region } \\
\text { Economic } \\
\text { level }\end{array}$ & Study design & $\begin{array}{l}\text { No. Of } \\
\text { total } \\
\text { samples }\end{array}$ & $\begin{array}{l}\text { Assessment } \\
\text { method of } \\
\text { PPD }\end{array}$ & $\begin{array}{l}\text { PPD } \\
\text { assessment } \\
\text { time }\end{array}$ & $\begin{array}{l}\text { Assessment } \\
\text { method of } \\
\text { violence }\end{array}$ & $\begin{array}{l}\text { Occurrence } \\
\text { time of } \\
\text { violence }\end{array}$ & $\begin{array}{l}\text { Type of } \\
\text { violence }\end{array}$ & $\begin{array}{l}\text { Reported } \\
\text { OR }(95 \% \\
\text { CI) }\end{array}$ & $\begin{array}{l}\text { Whether the } \\
\text { confounding } \\
\text { factors were } \\
\text { adjusted }\end{array}$ & $\begin{array}{l}\text { Quality } \\
\text { scores }\end{array}$ \\
\hline $\begin{array}{l}\text { Meltzer-Brody } \\
\text { et al [39] } \\
2013\end{array}$ & $\begin{array}{l}\text { American } \\
\text { (America) } \\
\text { High } \\
\text { income }\end{array}$ & $\begin{array}{l}\text { Prospective } \\
\text { cohort }\end{array}$ & 187 & EPDS $\geq 11$ & at 6 weeks & SI & lifetime & Violence & $\begin{array}{l}3.76 \\
(1.46- \\
9.66)\end{array}$ & Adjusted & 8 \\
\hline $\begin{array}{l}\text { Milgrom et al } \\
\text { [35] } \\
2008\end{array}$ & $\begin{array}{l}\text { Australian } \\
\text { (Oceania) } \\
\text { High } \\
\text { income }\end{array}$ & $\begin{array}{l}\text { Prospective } \\
\text { cohort }\end{array}$ & 10,777 & EPDS $\geq 13$ & at 6 weeks & AQ & lifetime & Violence & $\begin{array}{l}3.12 \\
(2.67- \\
3.64)\end{array}$ & crude & 7 \\
\hline $\begin{array}{l}\text { Abdollahi et al } \\
\text { [27] } \\
2014\end{array}$ & $\begin{array}{l}\text { Iran(Asia) } \\
\text { Upper } \\
\text { middle } \\
\text { income }\end{array}$ & $\begin{array}{l}\text { Prospective } \\
\text { cohort }\end{array}$ & 2083 & EPDS $\geq 13$ & at 8 weeks & SQ & lifetime & Violence & $\begin{array}{l}1.11 \\
(1.06- \\
1.16)\end{array}$ & crude & 7 \\
\hline $\begin{array}{l}\text { Tachibana et al } \\
\text { [25] } \\
2015\end{array}$ & $\begin{array}{l}\text { Japan(Asia) } \\
\text { High } \\
\text { income }\end{array}$ & $\begin{array}{l}\text { Prospective } \\
\text { cohort }\end{array}$ & 1378 & EPDS $\geq 9$ & at 1 months & AQ & lifetime & $\begin{array}{l}\text { Childhood } \\
\text { abuse }\end{array}$ & $\begin{array}{l}1.23 \\
(0.78- \\
1.92)\end{array}$ & Adjusted & 7 \\
\hline $\begin{array}{l}\text { Seng et al [41] } \\
2013\end{array}$ & $\begin{array}{l}\text { American } \\
\text { (America) } \\
\text { High } \\
\text { income }\end{array}$ & $\begin{array}{l}\text { Prospective } \\
\text { cohort }\end{array}$ & 566 & PDSS & at 6 weeks & SQ & lifetime & $\begin{array}{l}\text { Childhood } \\
\text { abuse }\end{array}$ & $\begin{array}{l}1.50 \\
(0.90- \\
2.50)\end{array}$ & crude & 6 \\
\hline $\begin{array}{l}\text { McDonald et al } \\
\text { [31] } \\
2012\end{array}$ & $\begin{array}{l}\text { Canada } \\
\text { (America) } \\
\text { High } \\
\text { income }\end{array}$ & $\begin{array}{l}\text { Prospective } \\
\text { cohort }\end{array}$ & 1578 & EPDS $\geq 10$ & at 4 months & SQ & recent & Violence & $\begin{array}{l}1.98 \\
(1.30- \\
3.10)\end{array}$ & Adjusted & 7 \\
\hline $\begin{array}{l}\text { Ghosh et al } \\
\text { [43] } \\
2011\end{array}$ & $\begin{array}{l}\text { Australia } \\
\text { (Oceania) } \\
\text { High } \\
\text { income }\end{array}$ & $\begin{array}{l}\text { Prospective } \\
\text { cohort }\end{array}$ & 6000 & EPDS $\geq 13$ & at $4 \sim 7$ days & $\mathrm{AQ}$ & lifetime & Violence & $\begin{array}{l}1.16 \\
(1.03- \\
1.31)\end{array}$ & crude & 6 \\
\hline $\begin{array}{l}\text { Gottfried et al } \\
\text { [45] } \\
2015\end{array}$ & $\begin{array}{l}\text { Israel(Asia) } \\
\text { High } \\
\text { income }\end{array}$ & $\begin{array}{l}\text { Longitudinal } \\
\text { study }\end{array}$ & 300 & $\mathrm{BDI} \geq 12$ & $\begin{array}{l}\text { at } 1-6 \\
\text { months }\end{array}$ & SEQ & lifetime & $\begin{array}{l}\text { Sexual } \\
\text { violence }\end{array}$ & $\begin{array}{l}2.36 \\
(1.02- \\
5.47)\end{array}$ & Adjusted & 7 \\
\hline $\begin{array}{l}\text { Li yang et al } \\
\text { [34] } \\
2017\end{array}$ & $\begin{array}{l}\text { China(Asia) } \\
\text { Upper } \\
\text { middle } \\
\text { income }\end{array}$ & $\begin{array}{l}\text { Longitudinal } \\
\text { study }\end{array}$ & 276 & $\mathrm{EPDS} \geq 12$ & at 4 weeks & CTQ & lifetime & $\begin{array}{l}\text { Any type } \\
\text { of abuse }^{\text {a }} \\
\text { Physical } \\
\text { abuse }^{\mathrm{a}} \\
\text { Emotional } \\
\text { abuse }^{\mathrm{a}} \\
\text { Sexual } \\
\text { abuse }^{\mathrm{a}}\end{array}$ & $\begin{array}{l}2.47 \\
(1.66- \\
3.68) \\
0.94 \\
(0.20- \\
4.81) \\
1.12 \\
(0.22- \\
5.58) \\
2.42 \\
(0.64- \\
9.18)\end{array}$ & Adjusted & 7 \\
\hline $\begin{array}{l}\text { Malta et al [36] } \\
\quad 2012\end{array}$ & $\begin{array}{l}\text { Canada } \\
\text { (America) } \\
\text { High } \\
\text { income }\end{array}$ & $\begin{array}{l}\text { Prospective } \\
\text { cohort }\end{array}$ & 1319 & EPDS $\geq 10$ & at 4 months & SQ & lifetime & $\begin{array}{l}\text { Any } \\
\text { violence } \\
\text { Childhood } \\
\text { abuse }{ }^{\mathrm{a}} \\
\text { Intimate } \\
\text { partner } \\
\text { violence }^{\mathrm{b}} \\
\text { Other } \\
\text { violence }\end{array}$ & $\begin{array}{l}1.78 \\
(1.26- \\
2.50) \\
1.83 \\
(1.11- \\
2.99) \\
1.66 \\
(0.95- \\
2.90)\end{array}$ & Adjusted & 8 \\
\hline $\begin{array}{l}\text { Dennis et al } \\
\text { [48] } \\
2017\end{array}$ & $\begin{array}{l}\text { Canada } \\
\text { (America) } \\
\text { High } \\
\text { income }\end{array}$ & $\begin{array}{l}\text { Prospective } \\
\text { cohort }\end{array}$ & 748 & EPDS $\geq 13$ & $\begin{array}{l}\text { at } 16 \\
\text { months }\end{array}$ & AAS & recent & Violence & $\begin{array}{l}4.14 \\
(1.43- \\
12.03)\end{array}$ & Adjusted & 7 \\
\hline $\begin{array}{l}\text { Sheela et al } \\
\text { [24] } \\
2016\end{array}$ & $\begin{array}{l}\text { India(Asia) } \\
\text { Lower } \\
\text { middle } \\
\text { income }\end{array}$ & $\begin{array}{l}\text { Prospective } \\
\text { cohort }\end{array}$ & 1600 & $\mathrm{EPDS} \geq 13$ & at 4-7days & AQ & lifetime & $\begin{array}{l}\text { Domestic } \\
\text { violence }\end{array}$ & $\begin{array}{l}6.91 \\
(4.52- \\
10.57)\end{array}$ & crude & 7 \\
\hline $\begin{array}{l}\text { Records et al } \\
\text { [35] } \\
2009\end{array}$ & $\begin{array}{l}\text { American } \\
\text { (America) } \\
\text { High } \\
\text { income }\end{array}$ & $\begin{array}{l}\text { Longitudinal } \\
\text { study }\end{array}$ & 139 & EPDS $\geq 12$ & $\begin{array}{l}\text { at } 2-8 \\
\text { months }\end{array}$ & SVAWS & lifetime & Violence & $\begin{array}{l}4.81 \\
(2.62- \\
8.86)\end{array}$ & crude & 7 \\
\hline $\begin{array}{l}\text { Leung et al [17] } \\
\quad 2002\end{array}$ & $\begin{array}{l}\text { China(Asia) } \\
\text { Upper } \\
\text { middle } \\
\text { income }\end{array}$ & $\begin{array}{l}\text { Prospective } \\
\text { cohort }\end{array}$ & 694 & EPDS $\geq 10$ & at 6 weeks & AAS & recent & $\begin{array}{l}\text { Domestic } \\
\text { violence }\end{array}$ & $\begin{array}{l}2.94 \\
(1.58- \\
5.49)\end{array}$ & crude & 7 \\
\hline $\begin{array}{l}\text { Woolhouse } \\
\text { et al [42] } \\
2012\end{array}$ & $\begin{array}{l}\text { Australia } \\
\text { (Oceania) } \\
\text { High } \\
\text { income }\end{array}$ & $\begin{array}{l}\text { Prospective } \\
\text { cohort }\end{array}$ & 1259 & EPDS $\geq 13$ & $\begin{array}{l}\text { at } 12 \\
\text { months }\end{array}$ & CAS & recent & $\begin{array}{l}\text { Physical } \\
\text { violence }^{\mathrm{b}}\end{array}$ & $\begin{array}{l}3.94 \\
(2.44- \\
6.36)\end{array}$ & Adjusted & 8 \\
\hline $\begin{array}{l}\text { Sorbo et al [29] } \\
\quad 2014\end{array}$ & $\begin{array}{l}\text { Norway } \\
\text { (Europe) }\end{array}$ & $\begin{array}{l}\text { Prospective } \\
\text { cohort }\end{array}$ & 53,065 & $E D S \geq 6$ & at 6 months & AAS & recent & $\begin{array}{l}\text { Any adult } \\
\text { violence }\end{array}$ & $\begin{array}{l}1.80 \\
(1.70-\end{array}$ & Adjusted & 8 \\
\hline
\end{tabular}


Table 1 (Continued)

\begin{tabular}{|c|c|c|c|c|c|c|c|c|c|c|c|}
\hline $\begin{array}{l}\text { First author } \\
\text { (reference) } \\
\text { publication } \\
\text { year }\end{array}$ & $\begin{array}{l}\text { Geographic } \\
\text { region } \\
\text { Economic } \\
\text { level }\end{array}$ & Study design & $\begin{array}{l}\text { No. Of } \\
\text { total } \\
\text { samples }\end{array}$ & $\begin{array}{l}\text { Assessment } \\
\text { method of } \\
\text { PPD }\end{array}$ & $\begin{array}{l}\text { PPD } \\
\text { assessment } \\
\text { time }\end{array}$ & $\begin{array}{l}\text { Assessment } \\
\text { method of } \\
\text { violence }\end{array}$ & $\begin{array}{l}\text { Occurrence } \\
\text { time of } \\
\text { violence }\end{array}$ & $\begin{array}{l}\text { Type of } \\
\text { violence }\end{array}$ & $\begin{array}{l}\text { Reported } \\
\text { OR(95\% } \\
\mathrm{CI})\end{array}$ & $\begin{array}{l}\text { Whether the } \\
\text { confounding } \\
\text { factors were } \\
\text { adjusted }\end{array}$ & $\begin{array}{l}\text { Quality } \\
\text { scores }\end{array}$ \\
\hline & $\begin{array}{l}\text { High } \\
\text { income }\end{array}$ & & & & & & & $\begin{array}{l}\text { Physical } \\
\text { violence } \\
\text { Sexual } \\
\text { violence } \\
\text { Emotional } \\
\text { violence }\end{array}$ & $\begin{array}{l}1.90) \\
1.40 \\
(1.10- \\
1.80) \\
1.60 \\
(1.40- \\
2.00) \\
1.70 \\
(1.60- \\
1.90)\end{array}$ & & \\
\hline $\begin{array}{l}\text { Gaillard et al } \\
\text { [49] } \\
2014\end{array}$ & $\begin{array}{l}\text { France } \\
\text { (Europe) } \\
\text { High } \\
\text { income }\end{array}$ & $\begin{array}{l}\text { Prospective } \\
\text { cohort }\end{array}$ & 264 & $\mathrm{EPDS} \geq 12$ & $\begin{array}{l}\text { at } 6-8 \\
\text { weeks }\end{array}$ & AQ & lifetime & $\begin{array}{l}\text { Physical } \\
\text { violence }^{\text {b }}\end{array}$ & $\begin{array}{l}3.00 \\
(1.10- \\
8.60)\end{array}$ & crude & 7 \\
\hline $\begin{array}{l}\text { Janssen et al } \\
\text { [30] } \\
2012\end{array}$ & $\begin{array}{l}\text { Canada } \\
\text { (America) } \\
\text { High } \\
\text { income }\end{array}$ & $\begin{array}{l}\text { Retrospective } \\
\text { cohort }\end{array}$ & 73,701 & EPDS $\geq 13$ & $\begin{array}{l}\text { at } 5-10 \\
\text { months }\end{array}$ & SQ & recent & Violence & $\begin{array}{l}3.37 \\
(2.04- \\
5.57)^{\mathrm{c}}\end{array}$ & Adjusted & 8 \\
\hline $\begin{array}{l}\text { LaCoursiere } \\
\text { et al [52] } \\
2010\end{array}$ & $\begin{array}{l}\text { American } \\
\text { (America) } \\
\text { High } \\
\text { income }\end{array}$ & $\begin{array}{l}\text { Prospective } \\
\text { cohort }\end{array}$ & 1027 & $\mathrm{EPDS} \geq 12$ & $\begin{array}{l}\text { at } 6-8 \\
\text { weeks }\end{array}$ & SQ & recent & Violence & $\begin{array}{l}1.16 \\
(0.69- \\
1.96)\end{array}$ & Adjusted & 8 \\
\hline $\begin{array}{l}\text { Gartland et al } \\
\text { [23] } \\
2016\end{array}$ & $\begin{array}{l}\text { Australia } \\
\text { (Oceania) } \\
\text { High } \\
\text { income }\end{array}$ & $\begin{array}{l}\text { Prospective } \\
\text { cohort }\end{array}$ & 1507 & EPDS $\geq 13$ & $\begin{array}{l}\text { within } 1 \\
\text { year }\end{array}$ & CAS & lifetime & $\begin{array}{l}\text { Any } \\
\text { violence } \\
\text { Childhood } \\
\text { physical } \\
\text { abuse }^{\mathrm{a}} \\
\text { Childhood } \\
\text { sexual }^{\text {abuse }}{ }^{\mathrm{a}} \\
\text { Emotional } \\
\text { violence }^{\mathrm{b}}\end{array}$ & $\begin{array}{l}1.41 \\
(1.10- \\
1.82) \\
1.70 \\
(1.20- \\
2.50) \\
1.20 \\
(0.90- \\
1.80) \\
3.00 \\
(1.96- \\
4.60)\end{array}$ & Adjusted & 8 \\
\hline $\begin{array}{l}\text { Turkcapar et al } \\
\text { [51] } \\
2015\end{array}$ & $\begin{array}{l}\text { Turkish } \\
\text { (Asia) } \\
\text { Upper } \\
\text { middle } \\
\text { income }\end{array}$ & $\begin{array}{l}\text { Prospective } \\
\text { cohort }\end{array}$ & 540 & $E P D S \geq 13$ & $\begin{array}{l}\text { at } 6-8 \\
\text { weeks }\end{array}$ & SQ & recent & Violence & $\begin{array}{l}6.60 \\
(3.02- \\
12.15)\end{array}$ & Crude & 8 \\
\hline $\begin{array}{l}\text { Rogathi et al } \\
\text { [22] } \\
2017\end{array}$ & $\begin{array}{l}\text { Tanzania } \\
\text { (Africa) } \\
\text { Low income }\end{array}$ & $\begin{array}{l}\text { Prospective } \\
\text { cohort }\end{array}$ & 1013 & EPDS $\geq 13$ & at 40 days & SQ & recent & $\begin{array}{l}\text { Any } \\
\text { violence } \\
\text { Emotional } \\
\text { violence } \\
\text { Physical } \\
\text { violence } \\
\text { Sexual } \\
\text { violence }\end{array}$ & $\begin{array}{l}2.51 \\
(1.67- \\
3.76) \\
1.46 \\
(0.92- \\
2.30) \\
2.15 \\
(1.13- \\
4.11) \\
1.98 \\
(1.22- \\
3.23)\end{array}$ & Adjusted & 9 \\
\hline $\begin{array}{l}\text { Escriba-Aguir } \\
\text { et al [46] } \\
2013\end{array}$ & $\begin{array}{l}\text { South } \\
\text { African } \\
\text { (African) } \\
\text { Upper } \\
\text { middle } \\
\text { income }\end{array}$ & $\begin{array}{l}\text { Longitudinal } \\
\text { study }\end{array}$ & 914 & $E P D S \geq 11$ & $\begin{array}{l}\text { at } 5-12 \\
\text { months }\end{array}$ & AAS & recent & $\begin{array}{l}\text { Emotional } \\
\text { violence }^{b}\end{array}$ & $\begin{array}{l}4.11 \\
(1.23- \\
13.73)\end{array}$ & Adjusted & 8 \\
\hline $\begin{array}{l}\text { Budhathoki } \\
\text { et al [53] } \\
2012\end{array}$ & $\begin{array}{l}\text { Nepal (Asia) } \\
\text { Low income }\end{array}$ & $\begin{array}{l}\text { Prospective } \\
\text { cohort }\end{array}$ & 72 & $\mathrm{EPDS} \geq 13$ & at 6 weeks & SQ & recent & $\begin{array}{l}\text { Emotional } \\
\text { violence }^{b} \\
\text { Physical } \\
\text { Violence } \\
\text { Sexual } \\
\text { Violence }\end{array}$ & $\begin{array}{l}1.71 \\
(0.28- \\
4.90) \\
1.71 \\
(0.45- \\
6.48) \\
1.04 \\
(0.20- \\
5.55) \\
1.53 \\
(0.49- \\
2.71) \\
1.36 \\
(0.37- \\
5.05) \\
0.35\end{array}$ & Crude & 7 \\
\hline
\end{tabular}


Table 1 (Continued)

\begin{tabular}{|c|c|c|c|c|c|c|c|c|c|c|c|}
\hline $\begin{array}{l}\text { First author } \\
\text { (reference) } \\
\text { publication } \\
\text { year }\end{array}$ & $\begin{array}{l}\text { Geographic } \\
\text { region } \\
\text { Economic } \\
\text { level }\end{array}$ & Study design & $\begin{array}{l}\text { No. Of } \\
\text { total } \\
\text { samples }\end{array}$ & $\begin{array}{l}\text { Assessment } \\
\text { method of } \\
\text { PPD }\end{array}$ & $\begin{array}{l}\text { PPD } \\
\text { assessment } \\
\text { time }\end{array}$ & $\begin{array}{l}\text { Assessment } \\
\text { method of } \\
\text { violence }\end{array}$ & $\begin{array}{l}\text { Occurrence } \\
\text { time of } \\
\text { violence }\end{array}$ & $\begin{array}{l}\text { Type of } \\
\text { violence }\end{array}$ & $\begin{array}{l}\text { Reported } \\
\text { OR(95\% } \\
\text { CI) }\end{array}$ & $\begin{array}{l}\text { Whether the } \\
\text { confounding } \\
\text { factors were } \\
\text { adjusted }\end{array}$ & $\begin{array}{l}\text { Quality } \\
\text { scores }\end{array}$ \\
\hline & & & & & & & & & $\begin{array}{l}(0.04- \\
2.98)\end{array}$ & & \\
\hline $\begin{array}{l}\text { Woolhouse } \\
\text { et al [26] } \\
2015\end{array}$ & $\begin{array}{l}\text { Australia } \\
\text { (Oceania) } \\
\text { High } \\
\text { income }\end{array}$ & $\begin{array}{l}\text { Prospective } \\
\text { cohort }\end{array}$ & 1507 & $E P D S \geq 13$ & $\begin{array}{l}\text { within } 1 \\
\text { year }\end{array}$ & CAS & recent & $\begin{array}{l}\text { Intimate } \\
\text { partner } \\
\text { violence }\end{array}$ & $\begin{array}{l}0.75 \\
(0.40- \\
1.60)\end{array}$ & Adjusted & 8 \\
\hline $\begin{array}{l}\text { Ludermir et al } \\
\text { [15] } \\
2010\end{array}$ & $\begin{array}{l}\text { Brazil } \\
\text { (America) } \\
\text { Upper } \\
\text { middle } \\
\text { income }\end{array}$ & $\begin{array}{l}\text { Prospective } \\
\text { cohort }\end{array}$ & 1045 & $E P D S \geq 12$ & $\begin{array}{l}\text { at } 3-6 \\
\text { months }\end{array}$ & SQ & recent & $\begin{array}{l}\text { Any } \\
\text { violence }{ }^{b} \\
\text { Emotional } \\
\text { violence } \\
\text { alone }^{b}\end{array}$ & $\begin{array}{l}1.54 \\
(1.13- \\
2.10) \\
1.58 \\
(1.04- \\
2.39)\end{array}$ & Adjusted & 9 \\
\hline $\begin{array}{l}\text { Katon et al [28] } \\
\quad 2014\end{array}$ & $\begin{array}{l}\text { American } \\
\text { (America) } \\
\text { High } \\
\text { income }\end{array}$ & $\begin{array}{l}\text { Prospective } \\
\text { cohort }\end{array}$ & 1423 & PHQ-9 $\geq 10$ & at 6 weeks & AAS & recent & $\begin{array}{l}\text { Intimate } \\
\text { partner } \\
\text { violence }\end{array}$ & $\begin{array}{l}0.53 \\
(0.24- \\
1.13)\end{array}$ & Adjusted & 7 \\
\hline $\begin{array}{l}\text { Patel et al [44] } \\
2002\end{array}$ & $\begin{array}{l}\text { India(Asia) } \\
\text { Lower } \\
\text { middle } \\
\text { income }\end{array}$ & $\begin{array}{l}\text { Follow up } \\
\text { study }\end{array}$ & 270 & $E P D S \geq 12$ & $\begin{array}{l}\text { at } 6-8 \\
\text { weeks }\end{array}$ & SI & lifetime & $\begin{array}{l}\text { Intimate } \\
\text { partner } \\
\text { violence }\end{array}$ & $\begin{array}{l}2.32 \\
(1.65- \\
3.26)\end{array}$ & Crude & 6 \\
\hline $\begin{array}{l}\text { Valentine et al } \\
\text { [50] } \\
2011\end{array}$ & $\begin{array}{l}\text { American } \\
\text { (America) } \\
\text { High } \\
\text { income }\end{array}$ & $\begin{array}{l}\text { Prospective } \\
\text { cohort }\end{array}$ & 190 & $\mathrm{BDI}-\mathrm{FS} \geq 4$ & $\begin{array}{l}\text { at 3-13 } \\
\text { months }\end{array}$ & AAS & recent & $\begin{array}{l}\text { Intimate } \\
\text { partner } \\
\text { violence }\end{array}$ & $\begin{array}{l}1.70 \\
(0.83- \\
3.52)\end{array}$ & Crude & 8 \\
\hline $\begin{array}{l}\text { Flach et al [32] } \\
\qquad 2011\end{array}$ & $\begin{array}{l}\text { England } \\
\text { (Europe) } \\
\text { High } \\
\text { income }\end{array}$ & $\begin{array}{l}\text { Prospective } \\
\text { cohort }\end{array}$ & 11,429 & $E P D S \geq 13$ & at 8 weeks & $\mathrm{AQ}$ & recent & $\begin{array}{l}\text { Domestic } \\
\text { violence }\end{array}$ & $\begin{array}{l}1.29 \\
(1.02- \\
1.63)\end{array}$ & Adjusted & 7 \\
\hline $\begin{array}{l}\text { Dolatian et al } \\
\text { [16] } \\
2010\end{array}$ & $\begin{array}{l}\text { Iran(Asia) } \\
\text { Upper } \\
\text { middle } \\
\text { income }\end{array}$ & $\begin{array}{l}\text { Follow up } \\
\text { study }\end{array}$ & 240 & $E P D S \geq 10$ & $\begin{array}{l}\text { at } 2-6 \\
\text { weeks }\end{array}$ & SQ & recent & $\begin{array}{l}\text { Domestic } \\
\text { violence }\end{array}$ & $\begin{array}{l}3.30 \\
(2.10- \\
5.10)\end{array}$ & Crude & 7 \\
\hline $\begin{array}{l}\text { Gausia et al } \\
\text { [47] } \\
2009\end{array}$ & $\begin{array}{l}\text { Bangladesh } \\
\text { (Asia) } \\
\text { Lower } \\
\text { middle } \\
\text { income }\end{array}$ & $\begin{array}{l}\text { Prospective } \\
\text { cohort }\end{array}$ & 346 & $E P D S \geq 10$ & $\begin{array}{l}\text { at } 6-8 \\
\text { weeks }\end{array}$ & SQ & lifetime & $\begin{array}{l}\text { Domestic } \\
\text { violence }\end{array}$ & $\begin{array}{l}1.00 \\
(0.30- \\
3.90)\end{array}$ & Adjusted & 7 \\
\hline
\end{tabular}

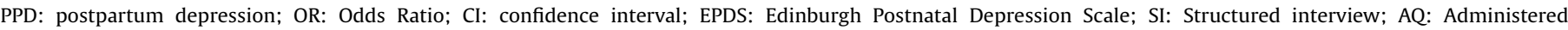

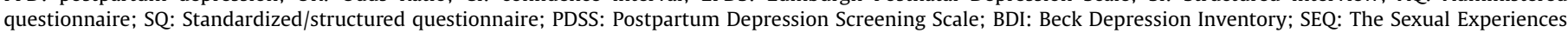

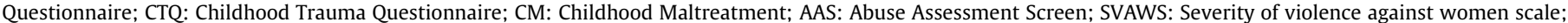
CAS: Composite Abuse Scale; PHQ-9: The Patient Health Questionnaire-9; BDI-FS: Beck Depression Inventory Fast Screen; ${ }^{\text {a }}$ Childhood abuse; ${ }^{\text {b }}$ Domestic violence.

Fig. 3 presents the results of pooled estimates between sexual, psychological and physical violence experiences and risk of developing PPD. Overall, the risk of developing PPD was significantly increased in women who experienced sexual ( $\mathrm{OR}=$ 1.56; 95\%CI: $1.35-1.81)$, emotional ( $\mathrm{OR}=1.75 ; 95 \% \mathrm{CI}$ : $1.61-1.89)$ and physical (OR=1.90; 95\%CI: 1.36-2.67) violence events. Nevertheless, there was substantial heterogeneity except for sexual violence experiences $\left(P=0.303 ; \mathrm{I}^{2}=17.1 \%\right)$.

Fig. 4displays the results of pooled estimates between domestic and childhood violence experiences and risk of developing PPD. Overall, women experiencing domestic violence events (OR=2.05; 95\%CI: $1.50-2.80)$ or childhood violence events $(\mathrm{OR}=1.59 ; 95 \% \mathrm{CI}: 1.34-1.88)$ had a significantly higher risk of developing PPD, when compared with those without any violence events. Yet, the evidence heterogeneity was observed for domestic violence experiences $(P=0.000$; $\left.I^{2}=85.4 \%\right)$

\subsection{Subgroup analyses}

Subgroup analyses for the association between any violence experiences and risk of developing PPD were summarized in Table 2. Overall, a significantly increased risk of developing PPD was found in most of subgroups. After subgroup analyses, whether the confounding factors were adjusted (test for subgroup differences [TSD]: $\mathrm{I}^{2}=65.6 \%$ ), assessment method of PPD (TSD: $I^{2}=57.8 \%$ ), economic levels (TSD: $I^{2}=42.9 \%$ ) quality score (TSD: $\mathrm{I}^{2}=20.7 \%$ ), and geographic region (TSD: $\mathrm{I}^{2}=19.0 \%$ ) were identified as the first five of the most relevant heterogeneity moderators. However, the risk of developing PPD was not statistically different across above-mentioned subgroup variables (TSD: all $P \geq 0.09$ ).

\subsection{Sensitivity analyses}

Sensitivity analyses were conducted to explore potential sources of heterogeneity in the association between maternal violence experiences and PPD, and to examine the influence of various exclusion criteria on the overall risk estimates. Exclusion of 3 studies that were considered of low methodological quality yielded similar results $(\mathrm{OR}=2.11 ; 95 \% \mathrm{CI}$ : $1.75-2.54)$, with substantial evidence of heterogeneity $\left(P<0.00001 ; I^{2}=94.0 \%\right)$. Further exclusion of 13 studies in which the confounding factors were not adjusted when assessing the risk of developing PPD associated with maternal violence experiences also yielded similar results $(\mathrm{OR}=1.79 ; 95 \% \mathrm{CI}: 1.52-2.11)$, yet heterogeneity was still present $(P$ $<0.00001 ; \mathrm{I}^{2}=72.0 \%$ ). Additionally, exclusion of any single study at a time did not materially alter the overall pooled estimates (Fig. 5). 


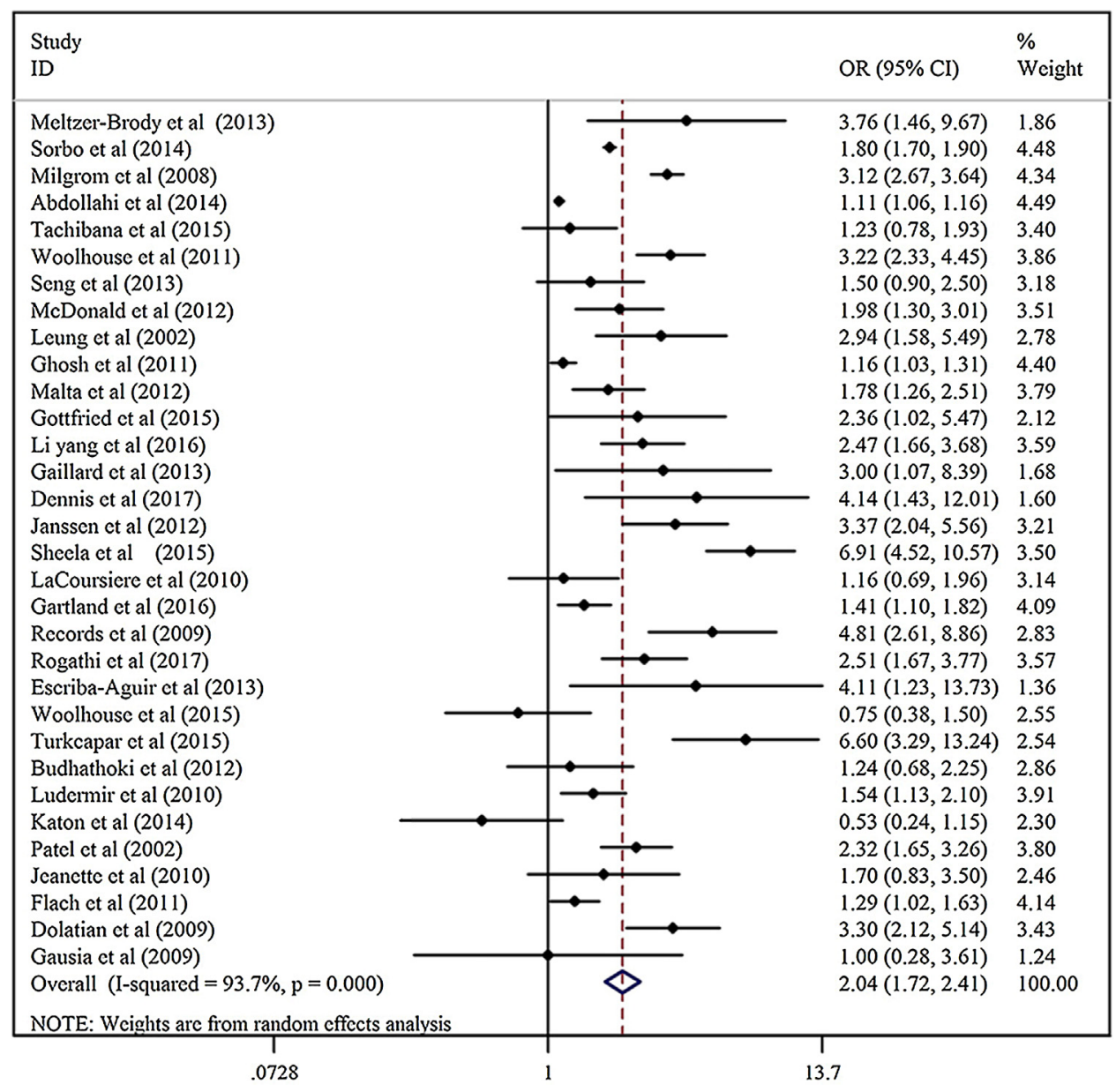

Fig. 2. Forest plot for maternal violence experiences and risk of PPD.

\subsection{Publication bias}

The Begg's funnel plot did show a little substantial asymmetry (Fig. 6), but the Begg's rank correlation test did not indicate the evidence of publication bias across studies of maternal violence experiences and risk of developing PPD $(P=0.709)$.

\section{Discussion}

PPD is an important part of the spectrum of mood disturbances affecting postpartum women, which can predispose to chronic or recurrent depression, and may affect the mother-infant relationship and child growth and development. Previous studies found that approximately half of new mothers may experience depressive symptoms at some point during the first year birth [48].

Our meta-analysis yielded following main findings. First, overall, maternal violence experiences were significantly associated with an increased risk of developing PPD (OR=2.04); second, different types of violence events such as sexual violence $(\mathrm{OR}=$ $1.56)$, emotional violence ( $O R=1.75)$, physical violence $(O R=1.90)$, domestic violence $(\mathrm{OR}=2.05)$ or childhood violence $(\mathrm{OR}=1.59)$ were also significantly associated with higher risks of developing PPD; and third, the association between maternal violence experiences and higher risks of developing PPD still existed after subgroup and sensitivity analyses, which indicated our results were stable and credible.

Until now, there are four existing reviews [18-21] that evaluated the association of maternal violence experiences with PPD. Our findings are generally consistent with previous reviews.
However, our study has important strengths. Our review is the most up to date on this subject. With the accumulating evidence and enlarged sample size, we have enhanced statistical power to provide more precise and reliable risk estimates. In our study, more than half of studies had a large sample size ( $>1000$ ); nearly $90 \%$ of studies were considered of higher methodological quality; and these high-quality studies contributed most of study participants. All included original studies used a cohort study design, which minimizes recall and selection biases. Moreover, compared with previous four meta-analyses, we fully considered the effect of geographic region, economic levels, assessment method of PPD, PPD assessment time, assessment method of violence, occurrence time of violence, whether the confounding factors were adjusted, and quality score. We found that the association between maternal violence experiences and risk of developing PPD persists and remains statistically significant after subgroup and sensitivity analyses. The most relevant heterogeneity moderators have been identified by subgroup analyses. Besides, our study indicated different types of violence experiences such as such as sexual, emotional and physical violence, or domestic or childhood violence, were significantly associated with PPD, which did not be confirmed by previous reviews.

To our knowledge, the underlying mechanisms involved in the association between violence and PPD are unclear. The most probable explanation is stress-related neuroendocrine dysfunctions. Evidence shows that the neuroendocrine channel comprising hypothalamic pituitary adrenal (HPA) axis responds to stress by hormonal secretion. The level a person experiences stress is directly related to the intensity of neuroendocrine response that 


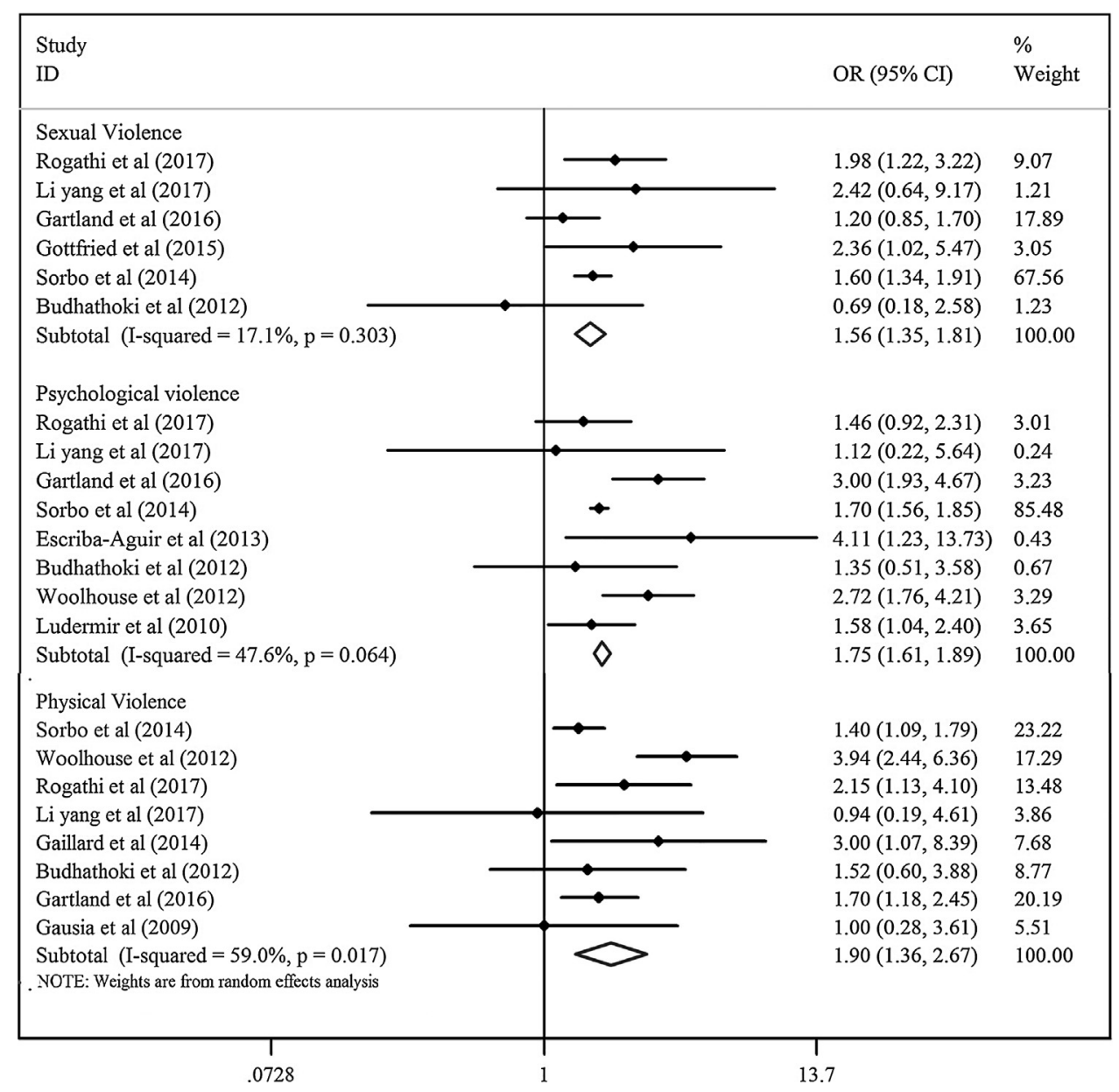

Fig. 3. Forest plot for sexual, psychological and physical violence experiences and risk of PPD.

promote the hormones secretion. Violence is a type of stress. When women experienced various forms of violence events in childhood, adolescence and adulthood, these adverse events could lead to a higher level of stress and activate the axis [1,16,35,54,55].

Additionally, there are a few possible explanations for the association between maternal violence experiences and risk of PPD. On the one hand, the association between violence and the risk of PPD may be influenced by cultural, social and behavior aspects of violence. Evidence indicates that violated women may be unwilling to disclose their violence experience because of the effect of self-concept, self-confidence, dignity, competence and even social stigma. Therefore, the expression of depression symptoms may be a reaction to violence and an indirect request for help. In addition, it is widely believed that violence is private matter and should be kept secret, which hinder women to seek for heal and lead to continuation of their problems $[16,35,56]$. On the other hand, they thought that who experienced violence events may lead to psychological trauma and psychological sensitivity, which brings about an increased risk of developing PPD [17]. In a word, the uncertainty of underlying mechanisms between violence and PPD warrants further research.

Substantial heterogeneity was observed among studies assessing the association of maternal violence experiences with risks of PPD, which was not surprising given the differences in study population and methodology. In our review, subgroup analyses were used to explore heterogeneity sources. Our subgroup analyses have identified main heterogeneity moderators, including whether the confounding factors were adjusted (TSD: $I^{2}=65.6 \%$ ), assessment method of PPD (TSD: $\mathrm{I}^{2}=57.8 \%$ ), economic levels (TSD:
$\mathrm{I}^{2}=42.9 \%$ ), quality score (TSD: $\mathrm{I}^{2}=20.7 \%$ ), and geographic region (TSD: $\mathrm{I}^{2}=19.0 \%$ ). However, there were not statistically significantly differences for risks of developing PPD across above-mentioned subgroups, which indicated the reasons leading to heterogeneity among studies may be more perplexing.

Potential limitations of this study should be considered. Firstly, there were some differences for measure methods of exposures and outcomes between studies, which may increase the likelihood of misclassification bias and measurement bias, thereby over- or underestimating the strength of the association. For example, in the present study, for the measure of PPD, nearly $87 \%$ of included studies used EPDS, while the remaining studies used other tools. Meanwhile, for the assessment of violence, most of included studies used standardized violence scale and questionnaires to collect the information of maternal violence experiences, while eight studies did not. In particular, when we collect the information of childhood abuse based on recollection of experiences of violence, recall bias was inevitable. Therefore, it is difficult for us to commit to the complete elimination of misclassification bias and measurement bias. But evidence indicated that the memories of violence experiences remain fairly accurate over a long period [57]. In addition, the assessment time of PPD and the occurrence time of violence were also different between studies. So it is difficult to precise classify because of the limitation of original data.

Secondly, the substantial heterogeneity among studies for the association between maternal violence experiences and risk of developing PPD were observed. Nevertheless, we were able to detect the major source of heterogeneity through subgroup and 


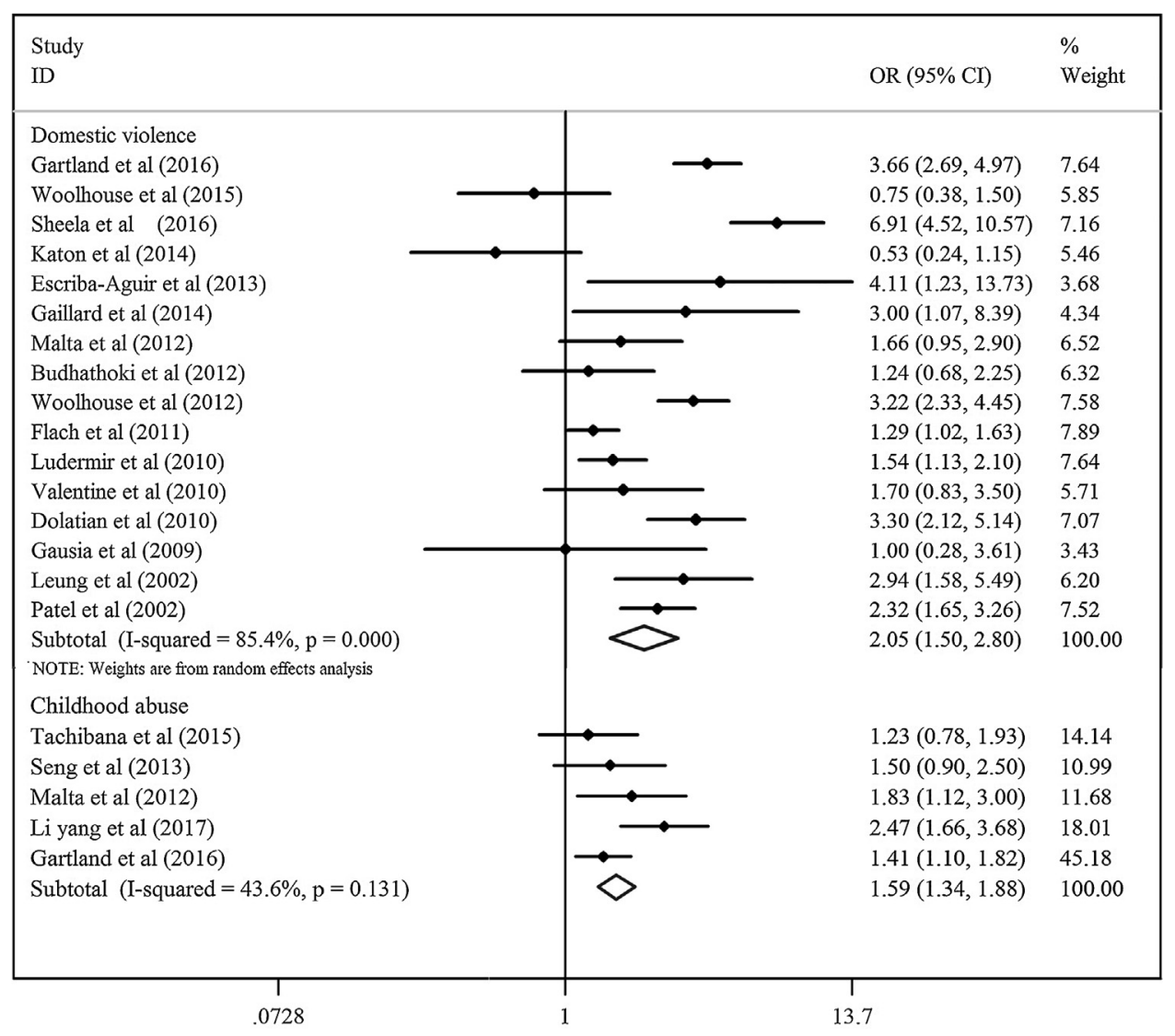

Fig. 4. Forest plot for domestic and childhood violence experiences and risk of PPD.

Table 2

Subgroup analysis of association between violence and the postpartum depression.

\begin{tabular}{|c|c|c|c|c|c|}
\hline \multirow[t]{2}{*}{ Subgroup variables } & \multirow[t]{2}{*}{ No.of studies } & \multirow[t]{2}{*}{ Pooled OR(95\%CI) } & \multicolumn{3}{|c|}{ Measure of heterogeneity } \\
\hline & & & $\chi^{2}$ & $P$ & $\mathrm{I}^{2}$ \\
\hline Continents & & & $4.94^{\mathrm{a}}$ & $0.29^{a}$ & $19.0 \%$ \\
\hline Asia & 11 & $2.35(1.49-3.70)$ & 157.19 & $<0.00001$ & $94.0 \%$ \\
\hline America & 9 & $1.73(1.27-2.35)$ & 25.83 & 0.001 & $69.0 \%$ \\
\hline Europe & 3 & $1.64(1.21-2.22)$ & 8.36 & 0.02 & $76.0 \%$ \\
\hline Oceania & 7 & $2.04(1.28-3.26)$ & 129.79 & $<0.00001$ & $95.0 \%$ \\
\hline Africa & 2 & $2.64(1.80-3.88)$ & 0.58 & 0.45 & $0.0 \%$ \\
\hline Economic levels & & & $1.75^{\mathrm{a}}$ & $0.19^{\mathrm{a}}$ & $42.9 \%$ a \\
\hline middle and low income countries & 12 & $2.48(1.64-3.75)$ & 174.21 & $<0.00001$ & $94.0 \%$ \\
\hline high income countries & 20 & $1.83(1.51-2.21)$ & 166.42 & $<0.00001$ & $89.0 \%$ \\
\hline Assessment method of PPD & & & $2.37^{\mathrm{a}}$ & $0.12^{\mathrm{a}}$ & $57.8 \%$ \\
\hline EPDS & 28 & $2.13(1.79-2.55)$ & 481.42 & $<0.00001$ & $94.0 \%$ \\
\hline Others ${ }^{b}$ & 4 & $1.34(0.76-2.36)$ & 7.85 & 0.05 & $62.0 \%$ \\
\hline PPD assessment time & & & $0.19^{\mathrm{a}}$ & $0.66^{\mathrm{a}}$ & $0.0 \%^{\mathrm{a}}$ \\
\hline$\leq 2$ months postpartum & 20 & $2.08(1.61-2.69)$ & 338.57 & $<0.00001$ & $94.0 \%$ \\
\hline$>2$ months postpartum & 12 & $1.94(1.61-2.34)$ & 33.73 & 0.0004 & $67.0 \%$ \\
\hline Assessment method of violence & & & $0.39^{\mathrm{a}}$ & $0.82^{\mathrm{a}}$ & $0.0 \%^{\mathrm{a}}$ \\
\hline Administered questionnaire & 8 & $2.28(1.45-3.61)$ & 153.94 & $<0.00001$ & $95.0 \%$ \\
\hline Standardized/structured questionnaire or interview & 12 & $1.92(1.42-2.60)$ & 96.43 & $<0.00001$ & $89.0 \%$ \\
\hline Violence assessment scale & 12 & $2.04(1.57-2.65)$ & 50.67 & $<0.00001$ & $78.0 \%$ \\
\hline Occurrence time of violence & & & $0.10^{\mathrm{a}}$ & $0.75^{\mathrm{a}}$ & $0.0 \%^{\mathrm{a}}$ \\
\hline recent & 17 & $1.99(1.62-2.45)$ & 76.76 & $<0.00001$ & $79.0 \%$ \\
\hline lifetime & 15 & $2.10(1.58-2.80)$ & 282.84 & $<0.00001$ & $95.0 \%$ \\
\hline Whether the effects were adjusted & & & $2.91^{\mathrm{a}}$ & $0.09^{\mathrm{a}}$ & $65.6 \%$ \\
\hline Adjusted & 19 & $1.79(1.52-2.11)$ & 64.55 & $<0.00001$ & $72.0 \%$ \\
\hline Unadjusted & 13 & $2.47(1.77-3.45)$ & 307.39 & $<0.00001$ & $96.0 \%$ \\
\hline Quality score & & & $1.26^{\mathrm{a}}$ & $0.26^{\mathrm{a}}$ & $20.7 \%^{\mathrm{a}}$ \\
\hline$<7$ & 3 & $1.57(0.97-2.54)$ & 14.85 & 0.0006 & $87.0 \%$ \\
\hline$\geq 7$ & 29 & $2.11(1.75-2.54)$ & 468.20 & $<0.00001$ & $94.0 \%$ \\
\hline
\end{tabular}

a Test for subgroup differences.

b includes Postpartum Depression Screening Scale, Beck Depression Inventory Fast Screen, Beck Depression Inventory and The Patient Health Questionnaire-9. 


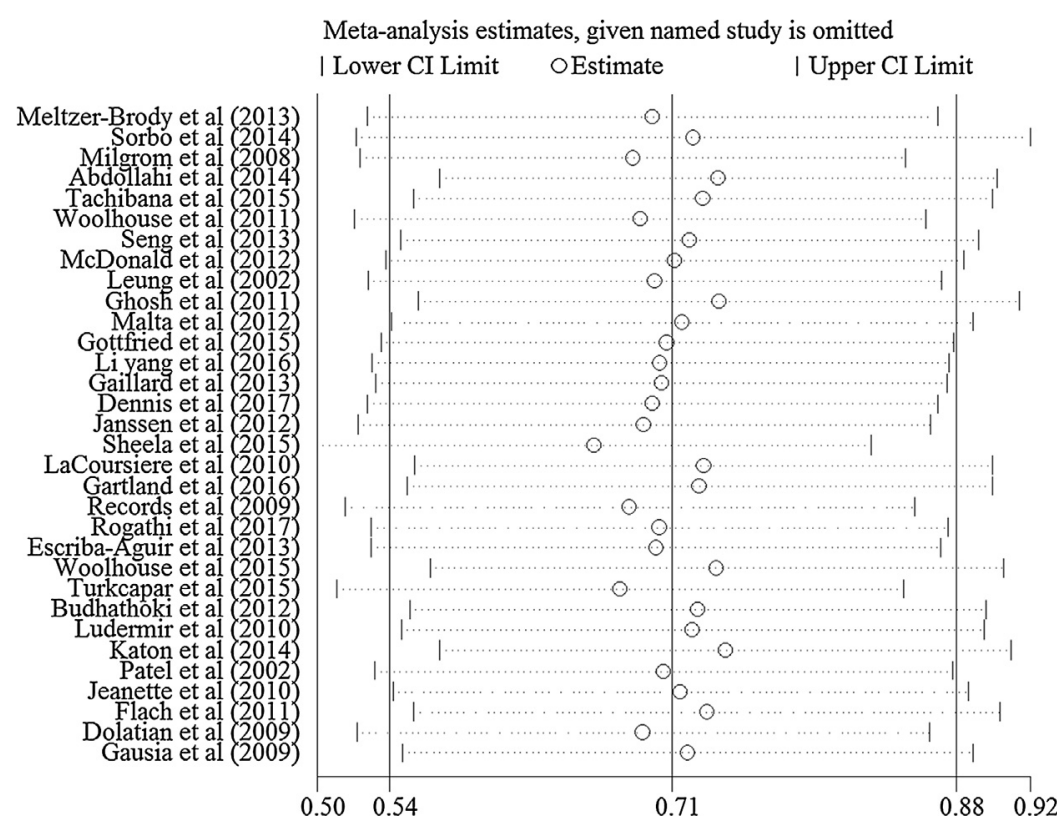

Fig. 5. Sensitivity analyses by excluding any single study at a time for maternal violence experiences and risk of PPD.

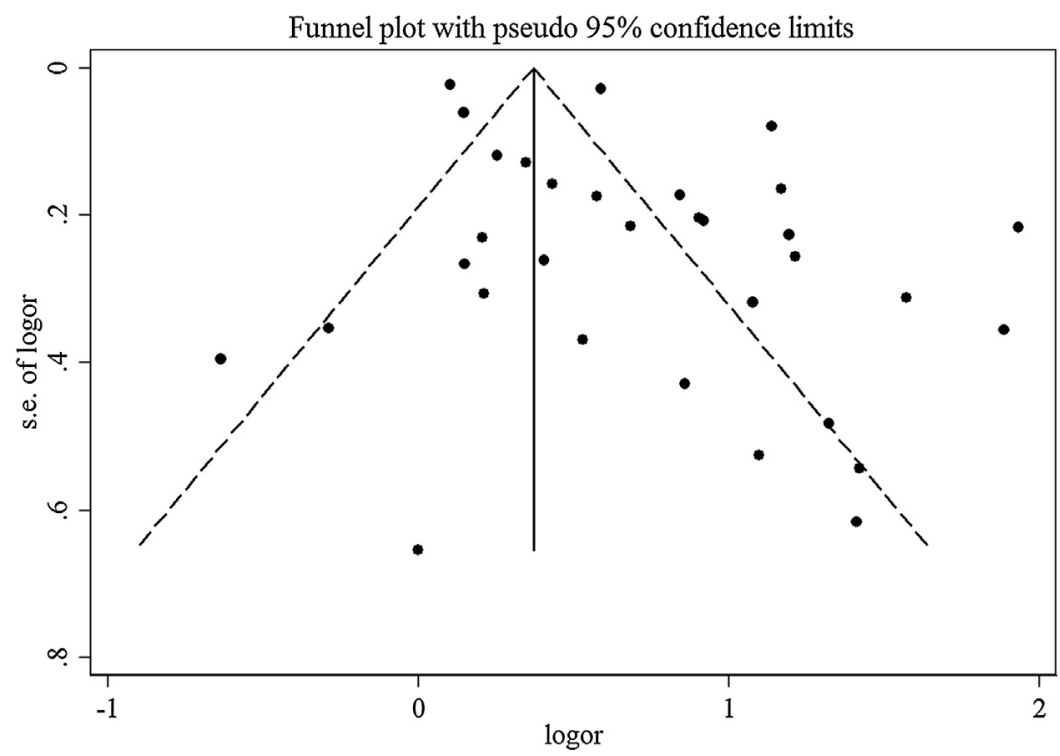

Fig. 6. Begg's funnel plot for maternal violence experiences and risk of PPD.

sensitivity analyses. The sensitivity analysis that omitted one study at a time and calculated the combined OR for the remaining studies yielded consistent results. After subgroup analysis, the heterogeneity was obviously decreased. However, our estimates have to be viewed with caution because of heterogeneity.

Thirdly, due to limited number of included studies on specific subtypes of violence, we did not perform a subgroup or sensitivity analysis for the association of specific violence events with PPD, so more studies should be included in future reviews, to provide further support for our results. Fourthly, residual confounding is of concern. Uncontrolled or unmeasured risk factors (such as responsibilities of perpetrators, laws, social condition, and the implications for health services or public health) potentially produce biases. Especially, cultural factors also are an important risk to the developing of PPD. Cultural diversity may vary between countries. However, we did not assess the influence of cultural factors on our outcome because the limitation of original data.
Although restricting analysis to studies that controlled conventional risk factors of PPD did not materially alter the combined risk estimates, we still cannot eliminate the possibility that residual confounding could affect the results because these factors do not explain all of the risk of PPD. Additionally, although the Begg's rank correlation test did not indicate the evidence of publication bias, Begg's funnel plot did show a little substantial asymmetry, which may influence the results. Finally, because our review only included studies published in Chinese or English, additional research in other populations is warranted to generalize the findings.

In conclusion, our study, which includes a large proportion of participants, with sufficient statistical power, aimed at providing an updated evidence to identify the risk of PPD associated with maternal violence experiences by conducting a meta-analysis of cohort studies. Although the role of potential bias and evidence of heterogeneity should be carefully evaluated, the present study 
indicated that women who experienced any violence events compared with those without experiencing any violence events, had a higher risk of developing PPD. Furthermore, it should be noted that specific subtypes of violence events such as sexual, emotional and physical violence, and domestic and childhood violence, were also associated with an increased risk of developing PPD.

Therefore, a comprehensive law against violence should be enacted, popularized and implemented in different counties. Improving the social and economic status of women, combating gender discrimination, and providing legal, medical as well as psychological services for violated women are very necessary to reduce violence and its outcomes. Meanwhile, early postpartum depression screening also plays an important role in preventing postpartum depression. In addition, the mechanisms involved in the association between violence and PPD are unclear and require further study for elucidation.

\section{Disclosure of interests}

The authors declare that they have no conflicts of interest.

\section{Details of ethics approval}

Not required.

\section{Funding}

JBQ was supported by the Project Funded by Natural Science Foundation of Hunan Province (2018JJ2551), National Natural Science Foundation Program of China (81803313), Hunan Provincial Key Research and Development Program (2018SK2063).

\section{Acknowledgments}

The authors would like to thank the editors and reviewers for their suggestions.

\section{References}

[1] O’Hara M.W., McCabe JE. Postpartum depression: current status and future directions. Annu Rev Clin Psychol 2013;9:379-407.

[2] Norhayati MN, Nik Hazlina NH, Asrenee AR, Wan Emilin WMA. Magnitude and risk factors for postpartum symptoms: a literature review. J Affect Disord 2015; 175:34-52.

[3] Gao L, Chan SW, You L, Li X. Experiences of postpartum depression among firsttime mothers in mainland China. J Adv Nurs 2010;66:303-12.

[4] Xie R, Liao S, Xie H, Guo Y, Walker M, Wen SW. Infant sex, family support and postpartum depression in a Chinese cohort. J Epidemiol Commun Health 2011;65:722-6.

[5] Shrivastava SR, Shrivastava PS, Ramasamy J. Antenatal and postnatal depression: a public health perspective. J Neurosci Rural Pract 2015;6:116-9.

[6] Wisner KL, Sit DK, McShea MC, Rizzo DM, Zoretich RA, Hughes CL, et al. Onset timing, thoughts of self-harm, and diagnoses in postpartum women with screen-positive depression findings. Jama Psychiatry 2013:70:490-8.

[7] Sockol LE, Epperson CN, Barber JP. The relationship between materna attitudes and symptoms of depression and anxiety among pregnant and postpartum first-time mothers. Arch Women Ment Health 2014;17:199-212.

[8] Glavin K, Leahy-Warren P. Postnatal depression is a public health nursing issue: perspectives from Norway and Ireland. Nurs Res Pract 2013;2013:1-7.

[9] Patel M, Bailey RK, Jabeen S, Ali S, Barker NC, Osiezagha K. Postpartum depression: a review. J Health Care Poor Underserved 2012;23:534-42.

[10] Palumbo G, Mirabella F, Gigantesco A. Positive screening and risk factors for postpartum depression. Eur Psychiatry 2017;42:77-85.

[11] Silverman ME, Reichenberg A, Savitz DA, Cnattingius S, Lichtenstein P, Hultman CM, et al. The risk factors for postpartum depression: a populationbased study. Depress Anxiety 2017;34:178-87.

[12] Chen HL, Cai JY, Zha ML, Shen WQ. Prenatal smoking and postpartum depression: a meta-analysis. J Psychosom Obstet Gynaecol. 20181-20189.

[13] Xu H, Ding Y, Ma Y, Xin X, Zhang D. Cesarean section and risk of postpartum depression: a meta-analysis. J Psychosom Res 2017;97:118-26.

[14] Ross LE, Dennis C. The prevalence of postpartum depression among women with substance use, an abuse history, or chronic illness: a systematic review. J Womens Health 2002;2009(18):475-86.
[15] Ludermir AB, Lewis G, Valongueiro SA, de Araujo TV, Araya R. Violence against women by their intimate partner during pregnancy and postnatal depression: a prospective cohort study. Lancet 2010;376:903-10.

[16] Dolatian M, Hesami K, Shams J, Majd HA. Relationship between violence during pregnancy and postpartum depression. Iran Red Crescent Med J 2010;12:377-83.

[17] Leung WC, Kung F, Lam J, Leung TW, Ho PC. Domestic violence and postnatal depression in a Chinese community. Int J Gynecol Obstet 2002;79:159-66.

[18] Wu Q, Chen H, Xu X. Violence as a risk factor for postpartum depression in mothers: a meta-analysis. Arch Womens Ment Health 2012;15:107-14.

[19] Howard LM, Oram S, Galley H, Trevillion K, Feder G. Domestic violence and perinatal mental disorders: a systematic review and meta-analysis. PLoS Med 2013;10:e1001452.

[20] Upadhyay RP, Chowdhury R, Aslyeh S, Sarkar K, Singh SK, Sinha B, et al Postpartum depression in India: a systematic review and meta-analysis. Bull World Health Organ 2017;95:706-17.

[21] Beydoun HA, Beydoun MA, Kaufman JS, Lo B, Zonderman AB. Intimate partner violence against adult women and its association with major depressive disorder, depressive symptoms and postpartum depression: a systematic review and meta-analysis. Soc Sci Med 2012;75:959-75.

[22] Rogathi JJ, Manongi R, Mushi D, Rasch V, Sigalla GN, Gammeltoft T, et al Postpartum depression among women who have experienced intimate partner violence: a prospective cohort study at Moshi, Tanzania. J Affect Disord 2017;218:238-45.

[23] Gartland D, Woolhouse H, Giallo R, McDonald E, Hegarty K, Mensah F, et al. Vulnerability to intimate partner violence and poor mental health in the first 4-year postpartum among mothers reporting childhood abuse: an Australian pregnancy cohort study. Arch Womens Ment Health 2016;19:1091-100.

[24] Sheela CN, Venkatesh S. Screening for postnatal depression in a tertiary care hospital. J Obstet Gynecol India 2016;66:72-6.

[25] Tachibana Y, Koizumi T, Takehara K, Kakee N, Tsujii H, Mori R, et al. Antenatal risk factors of postpartum depression at 20 weeks gestation in a japanese sample: psychosocial perspectives from a cohort study in Tokyo. PLoS One 2015;10:e142410

[26] Woolhouse H, Gartland D, Mensah F, Brown SJ. Maternal depression from early pregnancy to 4 years postpartum in a prospective pregnancy cohort study: implications for primary health care. Bjog Int J Obstet Gynaecol 2015;122:31221.

[27] Abdollahi F, Rohani S, Sazlina GS, Zarghami M, Azhar MZ, Lye MS, et al. Biopsycho-socio-demographic and obstetric predictors of postpartum depression in pregnancy: a prospective cohort study. Iran J Psychiatry Behav Sci 2014;8:11.

[28] Katon W, Russo J, Gavin A. Predictors of postpartum depression. J Women Health $2014 \cdot 23: 753-9$.

[29] Sorbo MF, Grimstad H, Bjorngaard JH, Lukasse M, Schei B. Adult physical sexual, and emotional abuse and postpartum depression, a population based prospective study of 53,065 women in the Norwegian Mother and Child Cohort Study. BMC Preg Childbirth 2014;14:316.

[30] Janssen PA, Heaman MI, Urquia ML, O'Campo PJ, Thiessen KR. Risk factors for postpartum depression among abused and nonabused women. Am J Obstet Gynecol 2012:207:481-9.

[31] McDonald S, Wall J, Forbes K, Kingston D, Kehler H, Vekved M, et al Development of a prenatal psychosocial screening tool for post-partum depression and anxiety. Paediatr Perinat Epidemiol 2012;26:316-27.

[32] Flach C, Leese M, Heron J, Evans J, Feder G, Sharp D, et al. Antenatal domestic violence, maternal mental health and subsequent child behaviour: a cohort study. Bjog Int J Obstet Gynaecol 2011;118:1383-91.

[33] Moher D, Liberati A, Tetzlaff J, Altman DG. Preferred reporting items for systematic reviews and meta-analyses: the PRISMA statement. Int J Surg 2010;8:336-41.

[34] Li Y, Long Z, Cao D, Cao F. Maternal history of child maltreatment and maternal depression risk in the perinatal period: a longitudinal study. Child Abuse Neg] 2017;63:192-201

[35] Records K, Rice MJ. Lifetime physical and sexual abuse and the risk for depression symptoms in the first 8 months after birth. J Psychosom Obst Gyn. 2009;30:181-90.

[36] Malta LA, McDonald SW, Hegadoren KM, Weller CA, Tough SC. Influence of interpersonal violence on maternal anxiety, depression, stress and parenting morale in the early postpartum: a community based pregnancy cohort study. BMC Pregnancy Childbirth 2012;12:153.

[37] Higgins JP, Thompson SG, Deeks JJ, Altman DG. Measuring inconsistency in meta-analyses. BMJ 2003;327:557-60.

[38] Egger M, Davey SG, Schneider M, Minder C. Bias in meta-analysis detected by a simple, graphical test. BMJ 1997;315:629-34.

[39] Meltzer-Brody S, Bledsoe-Mansori SE, Johnson N, Killian C, Hamer RM, Jackson C, et al. A prospective study of perinatal depression and trauma history in pregnant minority adolescents. Am J Obstet Gynecol 2013;208:211.

[40] Milgrom J, Gemmill AW, Bilszta JL, Hayes B, Barnett B, Brooks J, et al. Antenatal risk factors for postnatal depression: a large prospective study. J Affect Disord 2008; $108: 147-57$

[41] Seng JS, Sperlich M, Low LK, Ronis DL, Muzik M, Liberzon I. Childhood abuse history, posttraumatic stress disorder, postpartum mental health, and bonding: a prospective cohort study. J Midwifery Womens Health 2013;58:57-68.

[42] Woolhouse H, Gartland D, Hegarty K, Donath S, Brown SJ. Depressive symptoms and intimate partner violence in the 12 months after childbirth: a prospective pregnancy cohort study. Bjog Int J Obstet Gynaecol 2012;119:31523. 
[43] Ghosh A, Goswami S. Evaluation of postpartum depression in a tertiary hospital. J Obstet Gynecol India 2011;61:528-30.

[44] Patel V, Rodrigues M, DeSouza N. Gender, poverty, and postnatal depression: a study of mothers in Goa, India. Am J Psychiatry 2002;159:43-7.

[45] Gottfried R, Lev-Wiesel R, Hallak M, Lang-Franco N. Inter-relationships between sexual abuse, female sexual function and childbirth. Midwifery 2015;31:1087-95.

[46] Escribà-Agüir V, Royo-Marqués $\mathrm{M}$, Artazcoz L, Romito P, Ruiz-Pérez Longitudinal study of depression and health status in pregnant women: incidence, course and predictive factors. Eur Arch Psy Clin Neurosci 2013;263:143-51.

[47] Gausia K, Fisher C, Ali M, Oosthuizen J. Magnitude and contributory factors of postnatal depression: a community-based cohort study from a rural subdistrict of Bangladesh. Psychol Med 2009;39:999.

[48] Dennis C, Merry L, Gagnon AJ. Postpartum depression risk factors among recent refugee, asylum-seeking, non-refugee immigrant, and Canadian-born women: results from a prospective cohort study. Soc Psychiatry Psychiatric Epidemiol 2017;52:411-22.

[49] Gaillard A, Le Strat Y, Mandelbrot L, Keita H, Dubertret C. Predictors of postpartum depression: prospective study of 264 women followed during pregnancy and postpartum. Psychiatry Res 2014;215:341-6.

[50] Valentine JM, Rodriguez MA, Lapeyrouse LM, Zhang M. Recent intimate partner violence as a prenatal predictor of maternal depression in the first year postpartum among Latinas. Arch Womens Ment Health 2011;14:135-43.
[51] Turkcapar AF, Kadığlu N, Aslan E, Tunc S, Zayıfoğlu M, Mollamahmutoğlu L. Sociodemographic and clinical features of postpartum depression among Turkish women: a prospective study. BMC Preg Childbirth 2015:15:

[52] LaCoursiere DY, Barrett-Connor E, O Hara MW, Hutton A, Varner MW. The association between prepregnancy obesity and screening positive for postpartum depression. Bjog Int J Obstet Gynaecol 2010;117:1011-8.

[53] Budhathoki N, Dahal M, Bhusal S, Ojha H, Pandey S, Basnet S. Violence against women by their husband and postpartum depression. J Nepal Health Res Counc 2012;10:176-80.

[54] Brummelte S, Galea LA. Depression during pregnancy and postpartum: contribution of stress and ovarian hormones. Prog Neuropsychopharmacol Biol Psychiatry 2010;34:766-76.

[55] Meltzer-Brody S. New insights into perinatal depression: pathogenesis and treatment during pregnancy and postpartum. Dialogues Clin Neurosci 2011;13:89-100.

[56] Varma D, Chandra PS, Thomas T, Carey MP. Intimate partner violence and sexual coercion among pregnant women in India: relationship with depression and post-traumatic stress disorder. J Affect Disord 2007;102:22735.

[57] Zou C, Andersen JP. Comparing the rates of early childhood victimization across sexual orientations: heterosexual, lesbian, gay, bisexual, and mostly heterosexual. PLoS One 2015;1:e139198. 